\title{
La opinión del alumnado sobre la asignatura Didáctica de la Lengua Española I en el Grado de Educación Primaria (Universidad de Granada): evaluación y planteamientos de mejora
}

Dimitrinka G. Níkleva

Universidad de Granada

Recibido: 9 noviembre 2011 / Aceptado: 16 enero 2012

ISSN: $1697-7467$

RESUMEN: Según la filosofía del proceso de Bolonia, los estudiantes se convierten en el centro de un nuevo paradigma de aprendizaje en el marco del proceso formativo, por lo que deberían participar de forma activa en la construcción del Espacio Europeo de Educación Superior. Esta participación debe extenderse a la creación de los currículos, a los procesos de garantía de la calidad, etc.

Después del primer año de la implantación de los nuevos Grados consideramos que es necesario conocer la valoración del alumnado. Por este motivo, hemos realizado esta investigación, partiendo de la voluntad de escuchar la voz de los estudiantes para ajustar nuestra labor docente a sus expectativas.

Para este estudio nos hemos planteado los siguientes objetivos:

1) Conocer la opinión y la valoración de los alumnos sobre la asignatura Didáctica de la Lengua Española I.

2) Estudiar los factores que influyen sobre el grado de satisfacción general con la asignatura.

3) Conocer la opinión del alumnado sobre la utilidad de las prácticas realizadas.

4) Conocer la opinión y la necesidad del alumnado en cuanto a la cantidad de contenidos de Lengua y contenidos de Didáctica.

5) Formular resultados que permitan establecer propuestas de mejora.

Los participantes en nuestro estudio han sido 108 alumnos de primer curso del Grado de Educación Primaria, curso académico 2010/2011, en la facultad de Ciencias de la Educación, Universidad de Granada.

Hemos aplicado un método estadístico cuantitativo, realizado con el programa SPSS 17 (Statistical Product and Service Solutions).

Palabras clave: cuestionario de opinión del alumnado, asignatura Didáctica de la Lengua Española I, Grado de Primaria, análisis con SPSS.

Students' opinion on the course Spanish Language Teaching I (Degree on Primary Education, University of Granada): evaluation of standards and proposals for improvement

ABSTRACT: According to Bologna's process, students are at the centre of the new teaching paradigm, and consequently, they should participate actively in the European Space for Higher Education. This participation must affect the elaboration of the curricula, processes for guaranteeing quality, etc. 
After the first year of implementing the new degrees, students' opinion is of paramount importance. For this reason, we conduct this research focused on students' voice in order to adjust our teaching praxis to their expectations.

With this study, we aim to:

1) Know students' opinion and evaluation on the course Spanish Language Teaching I.

2) Study the factors which affect students' general satisfaction on the course.

3) Know students' opinion on the assessment practices.

4) Know students' opinion on the contents devoted to language and those devoted to didactics.

5) Formulate results which allow proposals for improvement.

A total of 108 students in their first year of studies participated in this study (academic year 2010/2011, Faculty of Educational Studies, University of Granada).

We apply quantitative analysis to this research and we use the software SPSS (Statistical Product Service Solutions) for data analysis.

Keywords: students' opinion questionnaire, course Spanish Language Teaching I, degree on Primary Education, data analysis with SPSS.

\section{INTRODUCCIÓN}

La implantación de los nuevos Grados requiere un estudio de este primer curso 2010/2011 para que nos sirva de evaluación y una futura planificación de la docencia que contemple los aciertos y desaciertos con el objetivo último de mejorar todos los aspectos de nuestra labor docente.

En el Espacio Europeo de Educación Superior (EEES) los alumnos tienen voz y voto en muchas cuestiones que afectan su formación. Aprendemos todos juntos a asumir esta nueva faceta de nuestros alumnos como agentes que participan de forma activa en el proceso de su formación.

En estos tiempos de innovación docente, en los que el centro del proceso se traslada del "enseñar" al "aprender", es muy importante saber encontrar las metodologías más adecuadas y la planificación, en general, más acertada que consigan un aprendizaje de mayor calidad y eficacia.

Un principio básico de la calidad de la formación consiste en enfocar el proceso en el cliente. Esto significa que hay que conocer y analizar las opiniones y las valoraciones de los propios alumnos para mejorar la calidad. Por este motivo, nos hemos planteado como objetivo fundamental de esta investigación el de estudiar la opinión de nuestros alumnos sobre la asignatura Didáctica de la Lengua Española en el grado de Educación Primaria, impartida durante el curso académico 2010/2011.

\section{ESTUdIOS PRECEDENTES}

Los estudios precedentes son numerosos, puesto que el proceso de Bolonia y el Espacio Europeo de Educación Superior (EEES) han suscitado muchos interrogantes e inquietudes que han desembocado en numerosas investigaciones. Entre ellas, con mucha frecuencia se ha optado por recoger y analizar la opinión del alumnado. 
Según el último informe TRENDS, el 95\% de las universidades europeas han adoptado el EEES, promovido por el Proceso de Bolonia (Rincón Igea y González Geraldo, 2010). Este resultado ha sido precedido por el proceso de implantación piloto del Sistema Europeo de Transferencia de Créditos (ECTS), que ha introducido todos los cambios más relevantes en cuanto a aspectos organizativos y metodológicos.

El objetivo de mejorar la calidad de la enseñanza-aprendizaje se fundamenta en los pilares del EEES, conocidos también como los cinco sentidos del Proceso de Bolonia (González Geraldo, Trevitt, Fazey y Carter, 2009, citado por Rincón Igea y González Geraldo, 2010):

1) armonización de titulaciones

2) transparencia del proceso

3) uso de los ECTS

4) incremento de la movilidad

5) fomento de la competitividad del EEES

Los nuevos planteamientos del EEES se resumen en el proyecto Tuning Educational Structures in Europe en 2002 (2007), donde se señala que el proceso de enseñanza-aprendizaje se centra en el alumno. Este proyecto conlleva la implantación de un enfoque por "resultados de aprendizaje" o un modelo basado en competencias y habilidades, frente a un sistema tradicional orientado al input y centrado en el profesor (Sabater Sempere et al., 2009). En el proyecto Tuning II en 2004 (2007) se recogen los modelos de enseñanza más adecuados, las metodologías y actividades que mejor se adaptan al desarrollo de competencias y las líneas de evaluación.

De la bibliografía sobre este tema nos parece conveniente destacar, entre muchos otros, "Modalidades de enseñanza centradas en el desarrollo de competencias. Orientaciones para promover el cambio metodológico en el Espacio Europeo de Educación Superior" (De Miguel Díaz, 2005).

Otros estudios reflejan la opinión del personal universitario y su adaptación al EEES, incluyendo las metodologías que consideran aplicables: "Prácticas docentes en el marco del Espacio Europeo de Educación Superior" de Billón y Jano (2008).

Las nuevas metodologías se orientan a reducir las clases teóricas que normalmente coinciden con las clases magistrales. En los casos en los que no es conveniente sustituir la clase magistral, hay que renovarla y complementarla. Desde el EEES se recomienda mantener los mismos objetivos en cuanto a la adquisición de conocimientos, pero con un nuevo proceso formativo, centrado en la adquisición de competencias y en el aprendizaje mediante métodos que se basan en los trabajos prácticos, el aprendizaje cooperativo y el aprendizaje por tareas (Fidalgo, 2007). En otros términos, se trata de aprender a aprender y realizar aprendizajes significativos.

Investigaciones previas (Rincón Igea y González Geraldo, 2010) señalan que las propuestas de mejora de los alumnos consisten principalmente en sugerir una valoración más alta de los trabajos que realizan en la evaluación final. Las críticas suelen referirse a la carga de trabajo diario, a la acumulación de tareas simultáneamente en varias asignaturas, un inconveniente que se podría superar con una mejor planificación y coordinación entre el profesorado.

En cuanto a las metodologías y las nuevas tecnologías usadas por el profesorado, los alumnos consideran que existe un uso excesivo de las proyecciones con el programa Power Point, hasta tal punto que a veces "cuando no funciona el cañón, no hay clase" (Rincón Igea y González Geraldo, 2010). Por todo ello, algunos alumnos llegan a reclamar las buenas 
clases magistrales. De todas formas, queremos matizar que la clase magistral actualmente ha adquirido un carácter más interactivo en comparación con la enseñanza de hace unas décadas.

Otro tema que los alumnos comentan son los trabajos en grupo. Estos presentan para ellos varios inconvenientes: la dificultad de compaginar horarios para reunirse, las diferencias en el nivel de conocimientos, disponibilidad, actitud y dedicación entre los miembros del grupo.

Las ventajas y los inconvenientes del trabajo en grupo y del trabajo autónomo vienen sistematizados por muchos autores, entre ellos, por Carpio de los Pinos (2008).

En cuanto a la evaluación, ya hemos comentado que los alumnos se sienten agobiados por la sobrecarga de trabajos que no encuentran su reflejo adecuado en la nota final. Un alumno del estudio citado afirma que: "Sale más rentable la opción antigua porque no vas perdiendo nota en los trabajos pequeños" (Rincón Igea y González Geraldo, 2010).

La evaluación de calidad, según Carpio de los Pinos (2008), debería cumplir las siguientes funciones:

1) Diagnóstico: permite conocer las dificultades, los errores, los logros, etc.

2) Diálogo: reflexionar sobre el proceso de enseñanza-aprendizaje, implicando a los alumnos.

3) Comprensión: debe ser la principal característica de la evaluación. Consiste en saber interpretar el proceso de enseñanza-aprendizaje.

4) Retroalimentación: sirve para elaborar una reorientación del proceso de enseñanzaaprendizaje.

5) Aprendizaje: es la etapa en que profesores y alumnos detectan las competencias y los conocimientos adquiridos, así como la adecuación de los métodos usados, etc.

Según la filosofía del proceso de Bolonia, los estudiantes se han convertido en el centro de un nuevo paradigma de aprendizaje en el marco del proceso formativo, por lo que deberían participar de forma activa en la construcción del Espacio Europeo de Educación Superior. Esta participación debe extenderse a la creación de los currículos, a los procesos de garantía de la calidad, etc. Sin embargo, varios informes señalan su escasa participación en comparación con el contexto anglosajón o el nórdico. Por tanto, se considera que la participación es una de la competencias cívicas que los alumnos deben adquirir a través de una educación para la ciudadanía democrática (Michavila y Parejo, 2008).

Por todo ello, esperamos que este estudio nos sirva para escuchar la voz de los estudiantes y para ajustar nuestra labor docente a sus expectativas.

\section{ObJetivos de LA InVEstigación}

Con este estudio nos planteamos los siguientes objetivos:

1) Conocer la opinión y la valoración de los alumnos sobre la asignatura Didáctica de la Lengua Española I.

2) Estudiar los factores que influyen sobre el grado de satisfacción general con la asignatura.

3) Conocer la opinión del alumnado sobre la utilidad de las prácticas realizadas.

4) Conocer la opinión y la necesidad del alumnado en cuanto a la cantidad de contenidos de Lengua y contenidos de Didáctica.

5) Formular resultados que permitan establecer propuestas de mejora. 


\section{Metodología}

\subsection{Participantes}

Los participantes en nuestro estudio han sido 108 alumnos del Grado de Educación Primaria (2 grupos), $1^{\circ}$ curso, curso académico 2010/2011, en la facultad de Ciencias de la Educación, Universidad de Granada. Este curso académico ha sido el primero de Grado, por lo tanto, los alumnos son los primeros que cursan esta asignatura con este nuevo diseño y planificación que corresponden a los requisitos del Espacio Europeo de Educación Superior.

\subsection{Instrumento de la investigación}

Nuestro instrumento de investigación es un cuestionario compuesto por 21 ítems en el que se combinan preguntas cerradas y abiertas y esto nos permite obtener datos cuantitativos y cualitativos.

La recogida de datos de este cuestionario garantizó el anonimato de las personas que lo han respondido, lo que aseguró la confidencialidad de la información recabada.

El primer bloque de preguntas estuvo dedicado a datos de identificación, donde se pidió a los alumnos que indicasen su edad, sexo, curso en el que están matriculados y titulación que cursaban. El siguiente bloque estuvo centrado en el objeto de estudio y constó de dieciocho preguntas que se refieren específicamente a la asignatura. De estas 18 preguntas, 15 son cerradas y 3 son preguntas abiertas. Se puede consultar el cuestionario completo en el Anexo I. Asimismo, la guía de la asignatura evaluada con el cuestionario se puede consultar en: http://www.ugr.es/ didlen/docencia/programa asignaturas index.html.

En las 15 preguntas cerradas hemos aplicado una escala de Likert donde se le pide al alumno que valore su grado de acuerdo con cada una de las afirmaciones presentadas mediante una valoración del 1 al 5:

$1=$ muy en desacuerdo

$2=$ algo en desacuerdo

$3=$ ni acuerdo ni desacuerdo (indiferente)

$4=$ algo de acuerdo

5 = muy de acuerdo

Con la última pregunta abierta (P18) hemos querido salvar las posibles faltas u olvidos de algún aspecto importante y le hemos ofrecido a los alumnos que opinen sobre cualquier aspecto que no haya sido recogido en el cuestionario: Comenta cualquier aspecto relacionado con la asignatura si lo consideras relevante y no se ha tratado en las preguntas anteriores.

En cuanto a la fiabilidad y validez del cuestionario, hemos obtenido los siguientes datos. Para las preguntas P1-P15 el alfa de Cronbach tiene el valor de 0,814 lo que indica mucha consistencia interna entre los datos. Para las preguntas P1-P17 es de 0,779. En ambos casos los datos nos confirman la fiabilidad del cuestionario.

La validez de nuestro instrumento se comprueba con los valores de $\mathrm{KMO}(, 798)$ y el test de Bartlett $(, 000)$ para las preguntas P1-P15. 


\subsection{Métodos de análisis}

El análisis de datos se realizó con el programa estadístico SPSS 17 (Statistical Product and Service Solutions), el más usado en Ciencias Sociales. Se realizó el análisis descriptivo de las variables individuales mediante tablas de frecuencia, medidas de centralización y dispersión adecuadas, gráficos, etc. Para el estudio de la relación entre variables cualitativas se han empleado tablas de contingencia y contrastes de independencia mediante el estadístico chi-cuadrado. Asimismo, se aplicó la prueba exacta de Fisher cuando la muestra no cumplía las condiciones para que la aplicación del contraste chi-cuadrado fuera adecuada (estas condiciones exigen que los valores esperados de al menos el $80 \%$ de las celdas en una tabla de contingencia sean mayores de 5). Se utilizó un alfa del 5\% para evaluar la significación de los contrastes. En los casos donde los contrastes fueron significativos se midió la fuerza de asociación mediante el coeficiente $\mathrm{V}$ de Cramer (cuyo rango se encuentra entre 0 -independencia- y 1 -asociación perfecta-).

\section{Análisis y discusión de los resultados}

En la tabla 1 se expone la ficha de evaluación, realizada por la profesora, respecto al número de alumnos matriculados, número aproximado de alumnos que asisten a clase, número de alumnos presentados al examen, aprobados (A) y suspensos (susp.). Se observa también que el número de alumnos que no llegaron a examinarse en ninguna de las convocatorias es de 6 alumnos en el grupo $1 \mathrm{y}$ de 11 alumnos en el grupo 2.

Tabla 1. Información sobre los alumnos matriculados, asistencia, número de aprobados y suspensos.

\begin{tabular}{|c|c|c|c|c|c|c|c|c|}
\hline grupo & alumnos & $\begin{array}{c}\text { asistencia } \\
\left(\text { n. }{ }^{\circ} \text { aprox.) }\right.\end{array}$ & \multicolumn{3}{|c|}{ junio 2011 } & \multicolumn{3}{c|}{ septiembre 2011} \\
\hline & & & presentados & A & susp. & presentados & A & susp. \\
\hline 1 & 61 & $45-50$ & 53 & 26 & 27 & 21 & 14 & 7 \\
\hline 2 & 65 & $45-48$ & 60 & 24 & 36 & 25 & 16 & 9 \\
\hline
\end{tabular}

\subsection{Resultados del análisis descriptivo}

Respecto a las preguntas de identificación, el 93,5\% de los estudiantes encuestados respondieron no tener titulación universitaria previa, mientras que el $6,5 \%$ restante sí presentan titulación anterior. Las edades de los estudiantes estuvieron comprendidas entre los 18 y 36 años, con una edad media de 19,9 y una moda de 19. Cabe destacar también una leve mayoría de mujeres $(54,6 \%)$ frente a los hombres $(45,4 \%)$.

En cuanto a las preguntas de opinión, los porcentajes de cada respuesta y medidas de centralidad obtenidas se detallan en la tabla 2. 


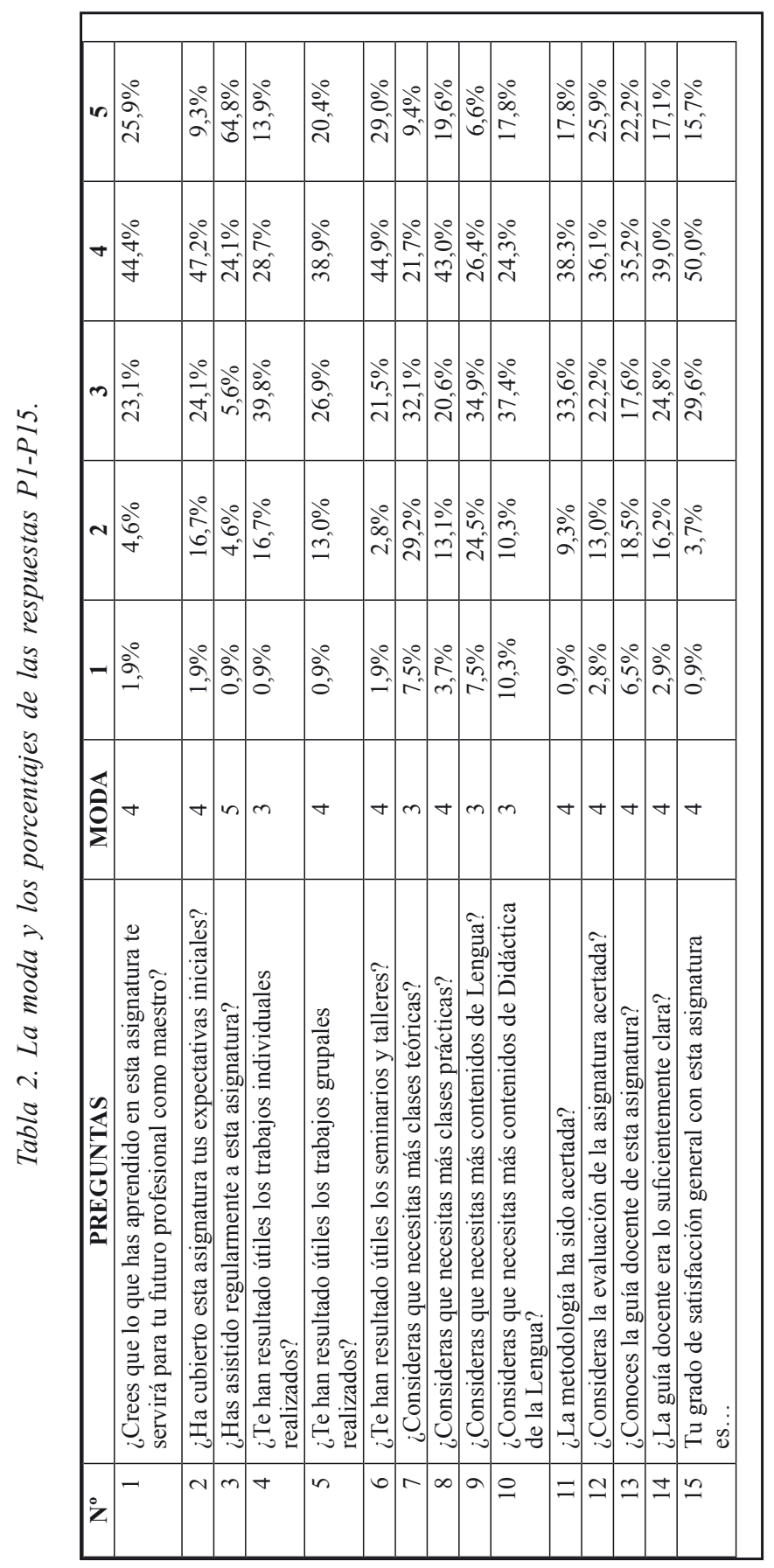


Observamos que el $44,4 \%$ de los alumnos consideran que lo que han aprendido en esta asignatura les servirá para su futuro profesional de maestros. El 47,2\% considera que la asignatura ha cubierto sus expectativas iniciales El 39,8\% consideran útiles los trabajos en grupo mientras que casi un $40 \%$ de los estudiantes se muestran indiferentes ante la utilidad de los trabajos autónomos. Asimismo, el mayor porcentaje de aceptación dentro de las actividades propuestas correspondió a los seminarios y talleres (45\%). A pesar de que la mayoría de los alumnos consideró acertada la metodología propuesta, un 62,6\% afirmó necesitar más clases prácticas de la asignatura impartida. Se observó una gran conformidad respecto a la evaluación de la asignatura, así como al manejo de la guía docente del curso. Por último, el $65,7 \%$ del alumnado se mostró satisfecho o muy satisfecho con la asignatura.

En cuanto a las preguntas abiertas, las tablas 3, 4 y 5 indican los tipos de respuesta reportados y sus porcentajes correspondientes. En la tabla 3 observamos que el 72,6\% de los alumnos han contestado que entre las prácticas les ha gustado más el trabajo en grupo sobre el análisis de libros de texto de Primaria, situando en segundo lugar los seminarios de gramática $(14,7 \%)$. Un $6,3 \%$ de los alumnos se mostraron a gusto con la totalidad de prácticas. Esta pregunta no ha sido respondida por 13 alumnos (12\%).

Tabla 3. Resultados de la P16: ¿Qué práctica te ha resultado más interesante?

\begin{tabular}{|c|l|c|}
\hline $\mathbf{N .}^{\mathbf{0}}$ & \multicolumn{1}{|c|}{ Práctica que más les ha gustado } & Porcentaje de alumnos \\
\hline 1 & en grupo: análisis de libros de texto de Primaria & $72,6 \%$ \\
\hline 2 & seminarios de gramática & $14,7 \%$ \\
\hline 3 & todas las prácticas & $6,3 \%$ \\
\hline 4 & corrección de una redacción & $2,1 \%$ \\
\hline 4 & resumen de una conferencia & $2,1 \%$ \\
\hline 5 & currículo y Real Decreto & $1,1 \%$ \\
\hline 5 & ninguna & $1,1 \%$ \\
\hline
\end{tabular}

Asimismo, la práctica menos valorada (tabla 4) ha sido el resumen de un artículo sobre las ciencias del lenguaje $(63,4 \%)$, seguida por la opinión de que todas las prácticas han sido interesantes $(11,3 \%)$ y en tercer lugar, por el análisis de libros de texto de Primaria $(9,9 \%)$. No han contestado 37 alumnos (34,3\%).

Tabla 4. Resultados de la P17: ¿Qué práctica te ha resultado menos interesante?

\begin{tabular}{|c|l|c|}
\hline $\mathbf{N .}^{\mathbf{o}}$ & \multicolumn{1}{|c|}{ Práctica que menos les ha gustado } & Porcentaje de alumnos \\
\hline 1 & resumen de un artículo (trabajo individual) & $63,4 \%$ \\
\hline 2 & todas han sido interesantes & $11,3 \%$ \\
\hline 3 & $\begin{array}{l}\text { análisis de libros de texto de Primaria (trabajo en } \\
\text { grupo) }\end{array}$ & $9,9 \%$ \\
\hline 4 & seminarios de gramática & $4,2 \%$ \\
\hline 4 & currículo y Real Decreto & $4,2 \%$ \\
\hline 4 & todas & $4,2 \%$ \\
\hline 5 & resumen de una conferencia (trabajo individual) & $1,4 \%$ \\
\hline 5 & corregir textos & $1,4 \%$ \\
\hline
\end{tabular}


La pregunta 18 es abierta: Comenta cualquier aspecto relacionado con la asignatura si lo consideras relevante y no se ha tratado en las preguntas anteriores. Pocos alumnos han respondido a la pregunta $18(15,7 \%$, tabla 5$)$, y varios alumnos han respondido reiterando afirmaciones de anteriores preguntas. Por ejemplo, repiten a menudo la pregunta 16 (la práctica que les ha resultado más interesante). En estos casos hemos ignorado la respuesta por ser repetitiva. Los que sí han contestado bien han aprovechado el espacio para opinar sobre la profesora o para formular algunas preferencias que tienen y que no han sido recogidas en las preguntas del cuestionario (Anexo II, algunas respuestas de la pregunta 18).

Tabla 5. Respuestas de la pregunta 18 (P18).

\begin{tabular}{|c|l|c|}
\hline $\mathbf{N}^{\mathbf{0}}$ & Tema & porcentaje de alumnos \\
\hline 1 & $\begin{array}{l}\text { trato excelente del profesor; motiva a los alumnos; } \\
\text { clases interesantes }\end{array}$ & 29,4 \\
\hline 1 & la asignatura debe ser más práctica & 29,4 \\
\hline 2 & necesita más seminarios de gramática & 11,8 \\
\hline 2 & la asistencia obligatoria es un problema & 11,8 \\
\hline 3 & todo perfecto & 5,9 \\
\hline 3 & prefiere grupos más reducidos & 5,9 \\
\hline 3 & más participación de los alumnos en clase & 5,9 \\
\hline
\end{tabular}

Nos parece oportuno añadir aquí la evaluación que ha recibido la profesora mediante un cuestionario de opinión del alumnado. La evaluación ha sido externa a la Universidad y se ha llevado a cabo por el Centro Andaluz de Prospectiva. La calificación obtenida fue del 4,45, para una calificación máxima posible de 5 puntos. La media de la titulación fue del $3,77 / 5,00$ y la media de la Universidad un $3,81 / 5,00$.

\subsection{Resultados del análisis inferencial}

Observamos que existe una asociación significativa entre el grado de satisfacción general del alumno con la asignatura y las variables asistencia, metodología aplicada, el sistema de evaluación, etc.

La asociación entre el grado de satisfacción general del alumno con la asignatura y la posesión de un título universitario anterior o la edad no fueron significativos.

Se confirmó una relación significativa entre el sexo y la satisfacción general $\left(x^{2}\right.$ $(4)=10,050, p<, 05$; test exacto de Fisher, $F=, 026)$. La fuerza de asociación, medida mediante el coeficiente V de Cramer, indicó una dependencia baja $(\mathrm{V}=, 305)$.

En la tabla 6 presentamos los resultados por frecuencia, según el sexo. 
Tabla 6. Satisfacción general con la asignatura (P15), según el sexo.

\begin{tabular}{|l|c|c|}
\hline \multicolumn{1}{|c|}{ Grado de satisfacción general } & \multicolumn{2}{c|}{ Sexo } \\
\hline muy poco & Mujer & Hombre \\
\hline poco & $0(0 \%)$ & $1(2 \%)$ \\
\hline bastante & $0(0 \%)$ & $4(8,2 \%)$ \\
\hline mucho & $19(32,2 \%)$ & $13(26,5 \%)$ \\
\hline excelente & $27(45,8 \%)$ & $27(55,1 \%)$ \\
\hline
\end{tabular}

Todas las preguntas de P1 a P14 presentan relación de dependencia con P15 (tabla 7). Esto significa que el grado de satisfacción general con la asignatura depende de las demás variables consideradas. 





La pregunta 16 (P16) sobre la práctica que les ha resultado más interesante a los alumnos no presenta relación de dependencia con el grado de satisfacción general $(\mathrm{F}=, 062, \mathrm{p}>, 05)$. Cabe destacar que no hemos podido aplicar el contraste de independencia Chi-cuadrado porque en 31 de las celdas $(83,6 \%)$ las frecuencias esperadas son menores que 5 , por lo que estos resultados no son fiables.

La pregunta sobre la práctica que ha resultado menos interesante (P17) tampoco presenta relación de dependencia con la satisfacción general $(\mathrm{F}=, 407, \mathrm{p}>, 05)$.

\section{Líneas de MEJORA del ESTUdio}

Después de nuestra experiencia con este estudio, hemos marcado varias líneas para mejorarlo en un futuro. En primer lugar, nos planteamos seguir mejorando la planificación de la asignatura, de acuerdo con los resultados obtenidos, y vamos a repetir el estudio en el segundo año de impartir la asignatura.

En el cuestionario añadiremos preguntas sobre:

1) El ambiente en clase (si ha sido agradable).

2) La utilidad de la guía docente.

3) La utilidad de las tutorías.

4) Propuestas de mejora.

En nuestro cuestionario no hemos planteado ninguna pregunta sobre las tutorías y creemos que hemos desaprovechado la oportunidad de conocer la opinión de los alumnos sobre este espacio. Las tutorías ofrecen muchas posibilidades de aplicar algunas de las novedades y los retos del EEES.

Consideramos muy importante el apartado de Propuestas de mejora, donde los alumnos podrán opinar libremente sobre cualquier aspecto. Algunos alumnos han aprovechado la pregunta 18 para hacerlo en el cuestionario actual (Comenta cualquier aspecto relacionado con la asignatura si lo consideras relevante y no se ha tratado en las preguntas anteriores). Sin embargo, con este nuevo enfoque de Propuestas de mejora creemos que obtendremos más opiniones y resultados que nos ayuden a seguir adaptando la asignatura a las necesidades de nuestros alumnos. Puesto que los alumnos suelen ignorar las preguntas de respuesta libre, conviene subdividir las Propuestas de mejora en varios subgrupos para que atiendan los siguientes aspectos:
1) teoría
2) prácticas
3) metodología
4) evaluación
5) ambiente en clase
6) tutorías
7) asistencia
8) otros

En el cuestionario actual hemos preguntado a nuestros alumnos sobre la práctica que les ha gustado más y la práctica que les ha resultado menos interesante. Sin embargo, opi- 
namos que probablemente los alumnos no se acuerdan de todas las prácticas o confunden las prácticas que han tenido que entregar con los seminarios de clase y los talleres. Por ello, en un futuro cuestionario enumeraremos las prácticas realizadas para facilitarles en la comprensión de la pregunta.

\section{Conclusiones}

En la reforma actual de la Universidad uno de los aspectos básicos consiste en escuchar la voz de los estudiantes y trabajar con ellos en la construcción de un contexto universitario democrático.

Nuestro estudio nos permite aprovechar la experiencia de este primer curso de los nuevos Grados y nos aporta líneas de actuación para mejorar la programación didáctica y el rendimiento de los estudiantes. Hemos formulado resultados que permiten volver a planificar y realizar los ajustes necesarios y todas las modificaciones que den como resultado una mejora en la formación de los futuros maestros y una mayor satisfacción por parte de los alumnos y del profesorado.

En primer lugar, hay que tener presente que el buen aprendizaje, tal como se concibe actualmente, debe ser crítico y debe llevar a saber pensar, sentir y actuar. Nuestro objetivo será ayudar al alumno a ser un agente que construye su propio aprendizaje y guiarlo en la adquisición de competencias para su futura actividad profesional.

Después del estudio realizado, nos planteamos promover la reflexión y el método de proyectos como métodos de aprendizaje activo (Huber, 2008). Consideramos que hay que seguir mejorando el diseño didáctico de la clase magistral, el trabajo guiado, el trabajo autónomo, el trabajo en grupo y las tutorías. Opinamos que el trabajo por proyectos y la resolución de problemas pueden ser bastante atractivos para los alumnos.

Nuestros alumnos han valorado mejor los trabajos en grupo comparados con los trabajos autónomos. Por consiguiente, consideramos que debemos cambiar el diseño de las prácticas para el próximo curso. Habrá que cambiar el enfoque de lectura de fuentes bibliográficas. Los trabajos autónomos tienen que plantearse como trabajos por proyectos o de resolución de problemas. Nuestros alumnos recomiendan una mayor conexión entre teoría y práctica. Necesitan sentirse preparados para impartir clases. Por tanto, nuestro objetivo debe ser fortalecer la conexión entre teoría y práctica.

Sin embargo, consideramos que el enfoque orientado a la adquisición de competencias no debe excluir el cognitivista, basado en el aprendizaje de contenidos. Se trata de encontrar la combinación óptima de ambos y aplicar los métodos más adecuados para cada uno.

Para mejorar la satisfacción de los alumnos con la evaluación vamos a adoptar la postura expresada anteriormente, según la cual la evaluación debe cumplir las siguientes funciones: diagnóstico, diálogo, comprensión, retroalimentación y aprendizaje (Carpio de los Pinos, 2008).

Nuestro estudio no contemplaba los valores de los estudiantes. Sin embargo, consideramos muy importante hacer los ajustes necesarios que nos sugieren los resultados y, a la vez, tener presente la dimensión ética que tenemos en nuestra labor docente y desarrollar 
actitudes y valores críticos que fomenten la ciudadanía y la responsabilidad. Uno de nuestros principales objetivos debe ser la formación de ciudadanos comprometidos con la sociedad.

\section{REFERENCIAS BIBLIOGRÁficas}

Álvarez Rojo, V.; Padilla Carmona, M. ${ }^{a}$ T.; Rodríguez Santero, J.; Torres Gordillo, J. J. y Suárez Ortega, M. (2011). "Análisis de la participación del alumnado universitario en la evaluación de su aprendizaje", en Revista Española de Pedagogía, 250.

Arumí Ribas, M. (2009). "Estudio de las percepciones de los estudiantes de lenguas extranjeras sobre el uso de instrumentos de autorregulación”, en Revista Española de Lingüística Aplicada (RESLA), 22: 35-58.

Billón, M. y Jano, M. (2008). Prácticas docentes en el marco del Espacio Europeo de Educación Superior. Madrid: Facultad de Ciencias Económicas y Empresariales de la Universidad Autónoma de Madrid.

Carpio de los Pinos, C. (2008). "Métodos de enseñanza-aprendizaje aplicables en Magisterio en el marco del Espacio Europeo de Educación Superior”, en Docencia e Investigación: revista de la Escuela Universitaria de Magisterio de Toledo, 33 (18).

De Miguel Díaz, M.; Alfaro Rocher, I. J.; Apodaca Urquijo, P. M.; Arias Blanco, J. M. García Jiménez, E.; Lobato Fraile, C. y Pérez Boullosa, A. (2006). Metodologías de Enseñanza y Aprendizaje para el Desarrollo de Competencias. Orientaciones para el profesorado universitario ante el Espacio Europeo de Educación Superior. Madrid: Alianza Editorial.

Fidalgo, A. (2007). Reducir las clases teóricas un diagnóstico unánime, pero... ¿cómo reducirlas? Consulta: 28/09/2011. En: http://innovacioneducativa.wordpress.com/2007/06/08/reducirlas-clases-teoricas-un diagnosticounanime-pero-\%c2\%bfcomo-reducirlas/

Huber, G. (2008). "Aprendizaje activo y metodologías educativas", en Revista de Educación (Madrid), núm. extraordinario, 59-81.

Gónzalez Geraldo, J. L., Trevitt, C., Carter, S. Y Fazey. J. (2009). Realising practical gains in the Bologna Process third cycle: a case study of the impact of these policy changes. Comunicación presentada en el ECER 2009, European Conference on Educational Research. Universidad de Viena, Austria. (Citado por Rincón Igea y González Geraldo, 2010).

Gutiérrez Garcia, C.; Pérez Pueyo, A.; Pérez Gutiérrez, M. y Palacios Picos, A. (2011). "Percepciones de profesores y alumnos sobre la enseñanza, evaluación y desarrollo de competencias en estudios universitarios de formación de profesorado", en Cultura y Educación, 23 (4), 499-514.

López de Silanes, J. L. (2011). “Aprendizaje y conocimiento para innovar y emprender”, en Árbor, 187 (extra 3), 3-13.

Michavila, F. y Parejo, J. L. (2008). "Política de participación estudiantil en el proceso de Bolonia", en Revista de Educación, núm. extraordinario: 85-118.

Ministerio de Educación y Ciencia (2006). Propuestas para la renovación de las metodologías educativas en la universidad. Consultado en: http://www.mec.es/educa/ccuniv/html/metodologias/docu/PROPUESTA_RENOVACION.pdf

Rincón Igea, B. y González Geraldo, J. L. (2010): "La voz de los estudiantes en el EEES: valoraciones sobre la implantación de los ECTS en la UCLM", en Docencia e investigación, 20: 59-85.

Sabater Sempere, Tarí Gilló, Fernández Sánchez, Andreu Guerrero, de Juana Espinosa, González Ramírez, Manresa Marhuenda, Rienda García, Valdés Conca (2009): La opinión del alum- 
nado sobre la metodología ECTS. En Gómez Lucas y Grau Company (coords.), Propuestas de diseño, desarrollo e innovaciones curriculares y metodologías en el EEES (687-700). Alicante: Universidad de Alicante (editorial Marfil).

Tunning Educational Structures in Europe (2007). La contribución de las universidades al proceso de Bolonia. Consulta: 03/09/2011/: http://www.tuning.unideusto.org/tuningeu/index.php?o ption $=$ content\&task $=$ view \&id $=175$ 


\section{ANEXO I \\ CUESTIONARIO}

ESTUDIO DE LA OPINIÓN DEL ALUMNADO DEL GRADO DE PRIMARIA SOBRE LA ASIGNATURA DIDÁCTICA DE LA LENGUA ESPAÑOLA I

FACULTAD DE CIENCIAS DE LA EDUCACIÓN, UNIVERSIDAD DE GRANADA CURSO 2010-2011

\section{CONTESTA A LAS SIGUIENTES PREGUNTAS DE FORMA ANÓNIMA.}

1. Titulación universitaria anterior: sí $\square$ no $\square$

2. Edad

3. Sexo

Mujer

Hombre

Marca solo un número de 1 a 5 (de menor a mayor grado) para expresar el grado de acuerdo o desacuerdo con cada afirmación.

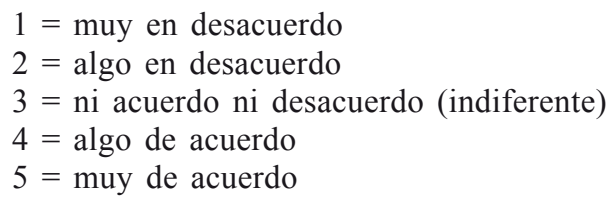

\begin{tabular}{|c|c|c|c|c|c|c|}
\hline $\mathbf{N}^{\mathbf{o}}$ & PREGUNTAS & \multicolumn{5}{|c|}{ de 1 a $5=$ de menor a mayor } \\
\hline 1 & $\begin{array}{l}\text { ¿Crees que lo que has aprendido en esta asignatura te servirá } \\
\text { para tu futuro profesional como maestro? }\end{array}$ & 1 & 2 & 3 & 4 & 5 \\
\hline 2 & ¿Ha cubierto esta asignatura tus expectativas iniciales? & 1 & 2 & 3 & 4 & 5 \\
\hline 3 & ¿Has asistido regularmente a esta asignatura? & 1 & 2 & 3 & 4 & 5 \\
\hline 4 & ¿Te han resultado útiles los trabajos individuales realizados? & 1 & 2 & 3 & 4 & 5 \\
\hline 5 & ¿Te han resultado útiles los trabajos grupales realizados? & 1 & 2 & 3 & 4 & 5 \\
\hline 6 & ¿Te han resultado útiles los seminarios y talleres? & 1 & 2 & 3 & 4 & 5 \\
\hline 7 & ¿Consideras que necesitas más clases teóricas? & 1 & 2 & 3 & 4 & 5 \\
\hline 8 & ¿Consideras que necesitas más clases prácticas? & 1 & 2 & 3 & 4 & 5 \\
\hline 9 & ¿Consideras que necesitas más contenidos de Lengua? & 1 & 2 & 3 & 4 & 5 \\
\hline 10 & $\begin{array}{l}\text { ¿Consideras que necesitas más contenidos de Didáctica de } \\
\text { la Lengua? }\end{array}$ & 1 & 2 & 3 & 4 & 5 \\
\hline 11 & ¿La metodología ha sido acertada? & 1 & 2 & 3 & 4 & 5 \\
\hline 12 & ¿Consideras la evaluación de la asignatura acertada? & 1 & 2 & 3 & 4 & 5 \\
\hline 13 & ¿Conoces la guía docente de esta asignatura? & 1 & 2 & 3 & 4 & 5 \\
\hline 14 & ¿La guía docente era lo suficientemente clara? & 1 & 2 & 3 & 4 & 5 \\
\hline 15 & Tu grado de satisfacción general con esta asignatura es... & 1 & 2 & 3 & 4 & 5 \\
\hline 16 & ¿Qué práctica te ha resultado más interesante? & & & & & \\
\hline 17 & ¿Qué práctica te ha resultado menos interesante? & & & & & \\
\hline 18 & $\begin{array}{l}\text { Comenta cualquier aspecto relacionado con la asignatura si } \\
\text { lo consideras relevante y no se ha tratado en las preguntas } \\
\text { anteriores. }\end{array}$ & & & & & \\
\hline
\end{tabular}




\section{ANEXO II \\ ALGUNAS RESPUESTAS DE LA PREGUNTA 18}

Pregunta 18. Comenta cualquier aspecto relacionado con la asignatura si lo consideras relevante y no se ha tratado en las preguntas anteriores.

La pregunta ha sido contestada por solo 17 alumnos. A continuación recogemos algunas de las respuestas reproducidas literalmente, con los errores:

Sujeto 66:

Me ha gustado mucho el trato que usted nos ha dado y lo interesante de cómo impartía las clases. Nos ha motivado mucho. Enhorabuena y espero que siga así. ¡Gracias!

Sujeto 94:

Estoy satisfecho con la profesora.

Sujeto 97:

Gracias por todos esos momentos en los que nos has sacado una sonrisa con tus anécdotas y sobre todo gracias por ser una verdadera PROFESORA.

Sujeto 32:

El temario es ameno, fácil de comprender e impartir y el trato del profesorado es excelente.

Sujeto 103:

Sobre todo destacar la comprensión y cercanía de la profesora, alejándose del estereotipo de profesor serio, que no le importan sus alumnos y que no hace por entenderlos, lo cual es muy común entre el profesorado.

Sujeto 100:

Creo que no he adquirido los conocimientos básicos para de aquí tres años ponerme delante un grupo/clase y dar una buena clase para que el alumnado aprenda.

Por otra parte, X. es una muy buena profesora y sé que no tiene nada que ver con la programación y todos los temas burocráticos.

Sujeto 62:

Quizás he echado en falta más didáctica sobre la lengua. Cómo enseñar ante una clase de niños y niñas a leer, escribir, analizar oraciones... No obstante, soy consciente de que para esto hay que tener una base teórica y conocer en profundidad los temas a tratar y, por desgracia, hay graves carencias en este sentido, por lo que también se tienen que tener en cuenta. Si la asignatura fuera anual en lugar de cuatrimestral se podría profundizar más en didáctica. 